Check for updates

Cite this: RSC Adv., 2017, 7, 56621

Received 5th October 2017

Accepted 24th November 2017

DOI: $10.1039 / c 7 r a 10970 a$

rsc.li/rsc-advances

\section{Electrostatic attraction driven and shuttle-like morphology assisted enhancement for tumor uptake $\uparrow$}

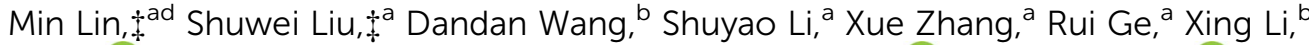 \\ Yi Liu, (DD a Wenzhi Song, ${ }^{* c}$ Hongchen Sun, *ab Hao Zhang (DD *a and Bai Yang (DD
}

Nanomaterials and nanocarriers have shown great potential in tumor theranostics, but low tumor uptake rate casts doubt on their practical applications due to competitive uptake by normal tissues and the immune system. In this work, we demonstrate the influence of electrostatic attraction and shuttle-like morphology on tumor uptake by designing positively charged $\mathrm{Cu}(॥)$ and Fe(II) doped polyaniline (CuPani) nanoshuttles (NSs). The experimental results indicate that such nanomaterials promote cellular adhesion/ internalization in vitro, and therefore enhance retention in tumor tissues. The CuPani NSs show electrostatic attraction driven nonspecific tumor recognition between oral epithelial carcinoma (KB) and Henrietta Lacks cancer (Hela) tumors. The uptake rate of CuPani NSs by negatively charged Hela tumors is up to $7.9 \%$ ID per $\mathrm{g}$ without any surface modification, while the uptake rate by KB tumors with a weaker charge is only $2.8 \%$ ID per $\mathrm{g}$. The high tumor uptake rate is attributed to the strong electrostatic attraction of CuPani NSs with tumor tissues, which is efficient when competing against clearance by the immune system. In addition, compared with stealth effect-matched nanoparticles, the NSs show an improved tumor uptake rate due to the one-dimension-like morphology for effective cellular internalization. The synergetic enhancement of tumor uptake by electrostatic attraction and shuttle-like morphology may be helpful in the design of novel nanodevices for performing tumor theranostics.

\section{Introduction}

Nanomaterials and nanocarriers have shown great potential in tumor theranostics. ${ }^{1-8}$ Plenty of nanodevices composed of multifunctional building blocks have been tested as agents to accomplish novel routes in tumor theranostics on the basis of tumor recognition, such as labelling, imaging and controlled drug delivery and release, as well as combined therapies. ${ }^{9-19}$ However, one of the major obstacles for the theranostic performance of nanodevices is low tumor uptake rate. ${ }^{20,21}$ Apart

${ }^{a}$ State Key Laboratory of Supramolecular Structure and Materials, College of Chemistry, Jilin University, Changchun 130012, P. R. China. E-mail: hao_zhang@ jlu.edu.cn

${ }^{b}$ Department of Oral Pathology, School and Hospital of Stomatology, Jilin University, Changchun 130021, P. R. China. E-mail: hcsun@jlu.edu.cn

'Department of Stomatology, China-Japan Union Hospital, Jilin University, Changchun 130031, P. R. China. E-mail: songwz@jlu.edu.cn

${ }^{d}$ Collaborative Innovation Center for Marine Biomass Fibers, Materials and Textiles of Shandong Province, Institute of Marine Biobased Materials, School of Materials Science and Engineering, Qingdao University, Qingdao 266071, China

$\dagger$ Electronic supplementary information (ESI) available: Additional XPS analysis, XRD, TEM, SEM, absorption spectra, cytotoxicity, zeta potential and confocal images as well as nonspecific adhesion in vitro. See DOI: 10.1039/c7ra10970a

\$ These authors contributed equally. from the targeting capability of nanodevices, the main concern is their weak interactions with proteins and cells in a complicated physiological environment, including van der Waals interactions, electrostatic interactions, hydrogen bonding, hydrophobic interactions and so forth. ${ }^{22}$ In general, most of the nanodevices entering the blood are quickly labelled by opsonizing proteins like immunoglobulin G (IgG) and complement factors as foreign substances, especially for positively charged nanodevices, and are then cleared out from the blood stream by the mononuclear phagocyte systems (MPSs) before an effective accumulation is formed in the tumor area. ${ }^{20}$ To avoid such an unspecific uptake by normal tissues and the MPS, great efforts are devoted to fabricating invisible covering shells that are capable of actively repelling their attachment to opsonizing proteins. ${ }^{2,9,20,23-26}$ The stealth effect improves the invisibility of nanodevices against the MPS and prolongs their circulation in the blood, thus improving the tumor uptake rate by virtue of the enhanced permeability and retention (EPR) effect of tumors. ${ }^{27}$

Resulting from the stealth effect, the long term accumulation of non-biodegradable nanomaterials in the body may raise safety concerns. Accordingly, the stealth effect of nanocarriers must be carefully controlled. ${ }^{20,28}$ This also means that the weak interactions between nanomaterials and bio-systems are not always harmful. Most recently, negatively charged nanocarriers 
were employed to load positively charged theranostic building blocks. Since most proteins carry negative charges, the nanocarriers with negatively charged surfaces are capable of avoiding attachment to labelling proteins due to electrostatic repulsion, which is favorable for longer circulation in the blood. The controlled release of positively charged building blocks near the tumor area will promote cellular adhesion/internalization, thus enhancing the tumor uptake rate. To design such nanocarriers, it is important to understand the weak interactions between nanocarriers and bio-systems, and balance the circulation of nanomaterials in the blood with their metabolization. This is still a challenge for most nanocarriers. ${ }^{3,29-33}$ Negatively charged polymers, such as polyethylene glycol (PEG) and polyphosphoester (PPE), are the most recognized materials for fabricating nanocarriers because they naturally possess longer blood circulation properties compared to inorganic nanoparticles (NPs). ${ }^{\mathbf{2 0 , 2 5 , 2 6 , 3 4 , 3 5}}$ After establishing active and/or passive targeting of cancerous cells/tumor tissues, the enhanced retention in the tumor tissues prohibits nanomaterials from returning to the blood and this compensates for the short circulation time caused by the fast clearance by the MPS in the blood. However, even after modification with specific targeting moieties, the tumor uptake rate of most nanomaterials is below $3 \%$ ID per $\mathrm{g}$, and after combinative modification with PEG the average uptake remains $\sim 5.5 \%$ ID per $g .{ }^{2}$ Since the low tumor uptake fails to compete with normal tissues and organs, such as the liver and kidneys, novel approaches for further enhancing the tumor uptake rate are greatly welcome.

In addition to the surface charges, the morphology and size of nanomaterials also impact tumor uptake. ${ }^{2}$ The most recognized size range is 10 to $100 \mathrm{~nm}$, considering the liver capture of larger NPs and renal filtration of smaller NPs, as well as poor retention in tumor tissues. ${ }^{3}$ However, efficient tumor accumulation requires both good penetration and substantial retention. In order to balance penetration and retention, an approach for directing small building blocks to penetrate into tumor tissues and then to aggregate into larger NPs in the tumor's acidic microenvironment has proven feasible for increasing the diffusion hindrance. ${ }^{3}$ However, it is still challenging for most nanomaterials with a stable conformation. In this context, the use of nanomaterials with a non-spherical morphology is considered to provide an alternative approach. Nanocarriers with a one-dimensional-like morphology may exhibit good retention in tumor tissues with a longitudinal dimension and improve the penetration in the transverse dimension, thus favoring cellular internalization and enhancing the efficiency in tumor theranostics.

In this work, $\mathrm{Cu}$ (II) and $\mathrm{Fe}(\mathrm{III})$ codoped polyaniline (CuPani) nanoshuttles (NSs) are tested as magnetic resonance imaging (MRI) nanoprobes for tumor diagnosis. The positively charged surface and shuttle-like morphology of the CuPani NSs lead to a fast cellular adhesion/internalization in vitro, which favors tumor uptake by enhancing the retention in the tumor tissues. Competing with uptake by the immune system, the current tumor uptake is electrostatic attraction driven. Because CuPani NSs have a stronger electrostatic attraction with Henrietta Lacks cancer (Hela) tumors than oral epithelial carcinoma (KB) tumors, the uptake rate by Hela tumors is up to $7.9 \%$ ID per $\mathrm{g}$, while it is only $2.8 \%$ ID per $g$ by KB tumors (Fig. 1). In comparison with stealth effect-matched nanoparticles, the NSs show an improved tumor uptake rate due to the one-dimensionlike morphology for effective cellular internalization.

\section{Experimental}

\subsection{Materials}

Sodium dodecyl sulfate (SDS, 99+\%), 3-(4,5-dimethyl-2-thiazolyl)-2,5-diphenyl-2- $H$-tetrazolium bromide (MTT), 2-mercaptoethylamine (MA, 99+\%), mercaptoglycerol and 3mercaptopropionic acid (MPA, 99+\%) were purchased from Sigma-Aldrich. Propidium iodide (PI) and fluorescein diacetate (FDA) were purchased from Invitrogen. Analytical grade copper chloride dihydrate $\left(\mathrm{CuCl}_{2} \cdot 2 \mathrm{H}_{2} \mathrm{O}\right)$, ferric chloride $\left(\mathrm{FeCl}_{3}\right)$, aniline, polyethylene glycol (PEG) and phosphate buffered saline (PBS) were all commercially available products and used as received without further purification. Deionized water was used in all experiments.

\subsection{Preparation of CuPani NSs and PEG-CuPani and SDS- CuPani NPs}

CuPani NSs were prepared according to our previous method. ${ }^{36}$ The reaction mixtures were prepared by sequential addition of $0.5 \mathrm{mmol}$ aniline, $1 \mathrm{mmol} \mathrm{CuCl}_{2}$ and $8 \mathrm{mmol} \mathrm{FeCl}_{3}$ in $120 \mathrm{~mL}$ water. As for the PEG-CuPani and SDS-CuPani NPs, $\mathrm{FeCl}_{3}$ is replaced by an initiator of $1 \mathrm{mmol}\left(\mathrm{NH}_{4}\right)_{2} \mathrm{~S}_{2} \mathrm{O}_{8}$ in the solution of PEG and SDS, separately. The mixtures were continuously stirred for $24 \mathrm{~h}$ at room temperature to perform polymerization. The products were collected by centrifugation at $8800 \mathrm{rpm}$ for $15 \mathrm{~min}$ and redissolved in water for further characterizations and tests.

\subsection{Adhesion of CuPani NPs on substrates}

Gold substrates were submerged in aqueous solution of MA and MPA with a concentration of $100 \mathrm{mM}$ for $24 \mathrm{~h}$ to obtain positively and negatively charged surfaces, respectively.

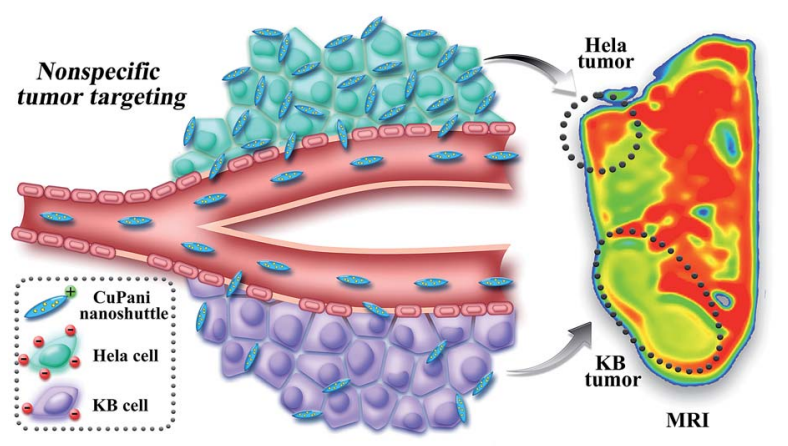

Fig. 1 Schematic illustration of tumor uptake affected by electrostatic attraction, also named nonspecific tumor recognition. The efficacy depends on the intensity of electrostatic attraction. Further assisted by the shuttle-like morphology, the enhanced retention in tumor tissues greatly elevates the performance in tumor diagnosis. 
Subsequently, the substrates were placed vertically into a solution of $1 \mathrm{mg} \mathrm{mL} \mathrm{m}^{-1}$ Pani and CuPani NSs. After $24 \mathrm{~h}$ incubation, the substrates were mildly washed, dried and observed by SEM. The quantities of NSs adhered on the substrates were evaluated by the reflective spectra. The reflective rate of empty gold substrates at $560 \mathrm{~nm}$ was set as $100 \%$ for estimating the reflective rate of the NP-treated substrates. The reduced reflective rate of the positively charged substrate treated by CuPani NPs was set as "1". Thus, the amounts of Pani and CuPani NPs adhered on the negatively charged surface were evaluated as “19.2" and "44.2", respectively.

\subsection{Nonspecific adhesion in vitro}

293, KB and Hela cells were firstly cultured in standard cell media. Subsequently, CuPani NSs were incubated with cells at different concentrations for $10 \mathrm{~min}$ in a 96-well plate (3000 cells per well). Then the cells were washed with saline 3 times to exclude excessive NSs. The quantities of adhered NSs were evaluated through optical density (OD) at $490 \mathrm{~nm}$. PEG-CuPani and SDS-CuPani NP experiments were also performed under the same conditions. Each experiment was repeated 5 times.

\subsection{Animal experiments and MRI}

30 4-6 week old BALB/c-nu mice were purchased from Beijing Huafukang Biological Technology Co. Ltd., and used under protocols approved by the Jilin University Laboratory Animal Center. After one week's observation, $2 \times 10^{6} \mathrm{~KB}$ and Hela cells were dispersed in $150 \mu \mathrm{L}$ cell culture media and injected subcutaneously into each side of the back legs. After 20 days of incubation, the mice were divided into six groups: the control, CuPani NSs, neutral NSs, negatively charged NSs and PEGCuPani and SDS-CuPani NPs groups. The control group consisted of mice i.v. injected with $100 \mu \mathrm{L}$ of saline while the other groups were separately injected with $100 \mu \mathrm{L} 2 \mathrm{mg} \mathrm{mL}^{-1}$ CuPani NSs, neutral NSs, negatively charged NSs and PEG-CuPani and SDS-CuPani NPs $(\sim 0.001 \%$ of the body weight $)$ in saline. MRI tests were carried out $24 \mathrm{~h}$ post injection under a $1.5 \mathrm{~T}$ magnetic field. The blood samples were collected by cutting their tails at different time intervals. Vital organs and tumors were taken by surgery $24 \mathrm{~h}$ post injection. Cu(II) was used to evaluate the content of CuPani by ICP-AES.

\subsection{Live subject statement}

This study was performed in strict accordance with the NIH guidelines for the care and use of laboratory animals (NIH Publication No. 85-23 Rev. 1985) and was approved by the Institutional Animal Care and Use Committee of National Tissue Engineering Center (Shanghai, China).

\subsection{Characterization}

UV-visible absorption spectra were obtained using a UV-3600 UV-vis spectrophotometer at room temperature under ambient conditions. Transmission electron microscopy (TEM) was conducted using a Hitachi $\mathrm{H}-800$ electron microscope at an acceleration voltage of $200 \mathrm{kV}$ with a CCD camera. X-ray photoelectron spectroscopy (XPS) was investigated using a VG ESCALAB MKII spectrometer with a $\mathrm{Mg} \mathrm{K} \alpha$ excitation $(1253.6 \mathrm{eV})$. Binding energy calibration was based on $\mathrm{C} 1 \mathrm{~s}$ at $284.6 \mathrm{eV}$. Scanning electron microscopy (SEM) images were acquired on a JEOL FESEM 6700F electron microscope. Atomic force microscope (AFM) tapping mode measurements were performed on a Nanoscope IIIa scanning probe microscope (Digital Instruments) using a rotated tapping mode etched silicon probe tip. Zeta potential measurements were performed using a Zetasizer NanoZS (Malvern Instruments). Fluorescent images of apoptotic cells were obtained by an Olympus IX71 inverted fluorescence microscope. Inductive coupled plasma emission spectrometry (ICP) was performed with a PERKIN ELMER OPTIMA 3300DV analyzer.

\section{Result and discussion}

\subsection{CuPani NSs and PEG- and SDS-CuPani NPs}

Positively charged CuPani NSs are prepared through surfactant free polymerization with a high concentration of $\mathrm{FeCl}_{3}$ as the oxidative initiator. From transmission electron microscopy
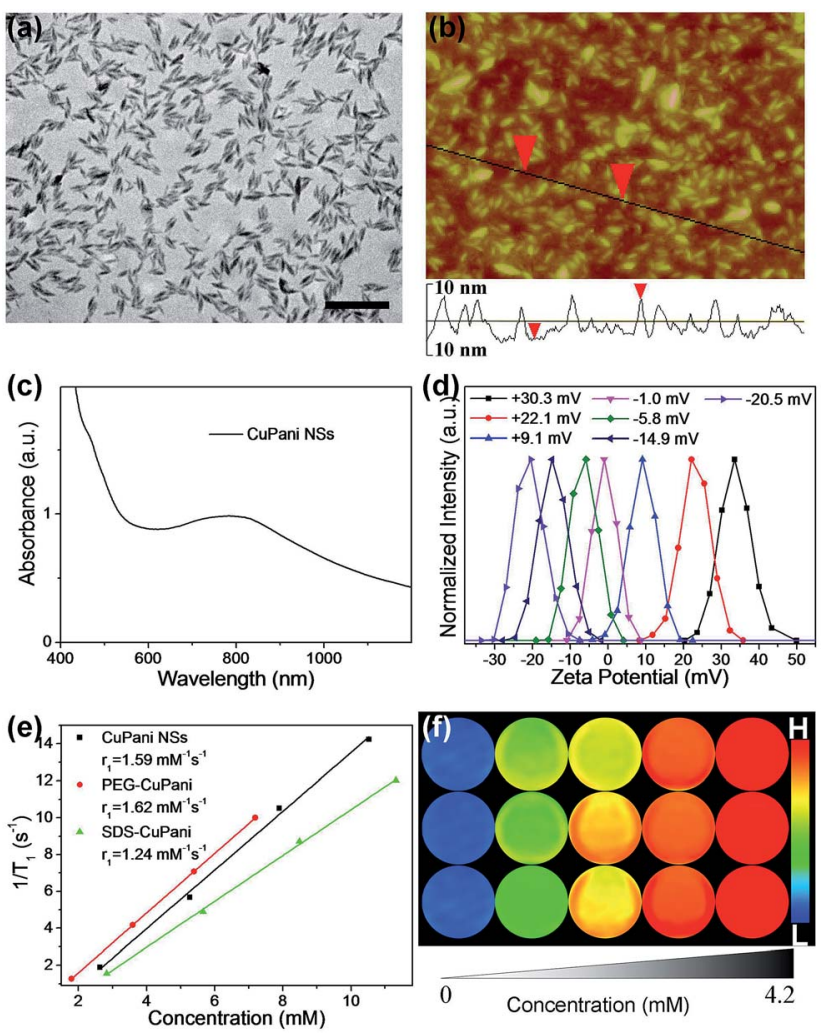

Fig. 2 Fundamental characterization of CuPani NSs. TEM image (a), AFM image (b) and UV-vis-NIR absorption spectra (c) of CuPani NSs. The scale bar represents $200 \mathrm{~nm}$. The surface potential of the CuPani NSs can be adjusted from positive to neutral and negative (d). The surface potentials of neutral and negatively charged NSs are set at -5.8 and $-20.5 \mathrm{mV}$, respectively. Concentration-dependent longitudinal relaxation $T_{1}(\mathrm{e})$ and $T_{1}$-weighted $M R I$ images (f) under a $1.5 \mathrm{~T}$ magnetic field for CuPani NSs and PEG-CuPani and SDS-CuPani NPs. The color bar stands for the MRI intensity from low (blue) to high (red). The concentration is based on the content of $\mathrm{N}$. 
(TEM) observations, the NSs are $62.6 \pm 13.4 \mathrm{~nm}$ in length and $14.1 \pm 3.9 \mathrm{~nm}$ in width (Fig. 2a). Additional atomic force microscopy (AFM) observations reveal the height to be $11.7 \pm$ $3.4 \mathrm{~nm}$ which is a bit lower than the width, probably due to gravity caused collapsing of the soft materials (Fig. $2 \mathrm{~b}$ ). $\mathrm{CuCl}_{2}$ is added before polymerization, and doped into Pani by virtue of coordinative interaction. As revealed by X-ray photoelectron spectroscopy (XPS) (Fig. S1 $\dagger$ ), the Cu spectrum exhibits the $\mathrm{Cu}-$ $\mathrm{Cl}, \mathrm{Cu}-\mathrm{O}$ and $\mathrm{Cu}-\mathrm{N}$ bonds at 934.6, 935.3, and $939.4 \mathrm{eV}$, respectively. ${ }^{37,38}$ The component of $\mathrm{O}$ is attributed to the hydration water of $\mathrm{Cu}(\mathrm{II})$. With respect to the $\mathrm{N}$ spectrum, the peaks at 398.3, 399.4, 400.1, 401.8 and $402.9 \mathrm{eV}$ represent the $\mathrm{N}-\mathrm{H}$ of amino, $\mathrm{N}-\mathrm{Cu}$, conjugated $\mathrm{N}=\mathrm{C}, \mathrm{N}-\mathrm{Fe}$ and $\mathrm{N}-\mathrm{H}$ of ammonium radical bonds, respectively (Fig. S1b †). ${ }^{39}$ The Fe spectrum shows the component of Fe(III) (Fig. S1c $\dagger$ ). ${ }^{40} \mathrm{Cl}$ is also determined as the counterion for $\mathrm{Cu}(\mathrm{II})$ and $\mathrm{Fe}(\mathrm{III})$, exhibiting binding energies of 198.9 and $200.5 \mathrm{eV}$ (Fig. S1d $\dagger$ ). ${ }^{38}$ The doping of metal cations leads to a strong absorption of CuPani NSs in the near-infrared (NIR) region due to the improved electron delivery between the quinoid and benzenoid rings (Fig. 2c). ${ }^{\mathbf{4 1}}$ Besides, the $\mathrm{Cu}$ (II) doping also increases the crystallization of polyaniline as revealed by XRD patterns (Fig. S2 $\dagger$ ). According to our previous study, the formula of CuPani NSs is speculated as $\left[\mathrm{C}_{6} \mathrm{H}_{5} \mathrm{~N}\left(\mathrm{CuCl}_{2}\right)_{0.38} \cdot\left(\mathrm{H}_{2} \mathrm{O}\right)_{0.23}\left(\mathrm{FeCl}_{3}\right)_{0.45}\right]_{n \cdot}{ }^{36}$

To evaluate the influence of electrostatic interactions on tumor uptake, the neutral and negative surface potentials of the CuPani NSs are achieved by adding sodium citrate (Fig. 2d). Besides, nearly neutralized PEG-modified CuPani and negatively charged SDS-modified CuPani NPs are also prepared by $\left(\mathrm{NH}_{4}\right)_{2} \mathrm{~S}_{2} \mathrm{O}_{8}$-initiated polymerization as controls (Fig. S3 and $\mathrm{S} 4 \dagger)$. The PEG-CuPani NPs are spherical with an average diameter of $81.6 \pm 13.1 \mathrm{~nm}$ (Fig. S3a $\dagger$ ). Similar to the XPS of the CuPani NSs, the PEG-CuPani NPs additionally show the $\mathrm{N}-\mathrm{H}$ bond at $401.2 \mathrm{eV}$, which is attributed to the hydrogen bonding between the $\mathrm{N}$ in Pani and the -OH in PEG (Fig. S1 and S3 $\dagger$ ). The SDS-CuPani NPs are amorphous and the XPS reveals additional binding at 935.4 and $936.5 \mathrm{eV}$ as $\mathrm{Cu}-\mathrm{O}$ from $\mathrm{SO}_{4}{ }^{2-}$, which is generated from the decomposed $\mathrm{S}_{2} \mathrm{O}_{8}{ }^{2-}$ and SDS (Fig. S4 $\dagger$ ). ${ }^{39}$ Moreover, because $\mathrm{Cu}$ (II) and $\mathrm{Fe}(\mathrm{III})$ possess single electrons in their $3 \mathrm{~d}$ orbits, the CuPani NSs and NPs are capable of shortening the longitudinal relaxation $\left(T_{1}\right)$ of protons. As determined by a $500 \mathrm{M}$ nuclear magnetic resonance (NMR) spectrometer, the relaxation rates $\left(r_{1}\right)$ for $\mathrm{Cu}(\mathrm{II})$ and $\mathrm{Fe}(\mathrm{III})$ are only 0.21 and $0.81 \mathrm{mM}^{-1} \mathrm{~s}^{-1}$, respectively (Fig. S5a $\dagger$ ), and the $r_{1}$ values for the CuPani NSs and the PEG-CuPani and SDS-CuPani NPs are calculated as 1.59, 1.62 and $1.24 \mathrm{mM}^{-1} \mathrm{~s}^{-1}$, respectively, based on the concentration of $\mathrm{N}$ (Fig. 2e), while they are 4.19, 2.91 and $3.52 \mathrm{mM}^{-1} \mathrm{~s}^{-1}$ when converted to the concentration of $\mathrm{Cu}$ (II) (Fig. S5b $\dagger$ ). Following further testing in a $1.5 \mathrm{~T}$ magnetic field, Fig. 2f shows the concentration-dependent $T_{1}$-weighted MRI images, confirming the potentials of the CuPani NSs and NPs as nanoprobes for MRI tests.

\subsection{Cellular adhesion/internalization in vitro}

Before further tests in vitro, the CuPani NSs and the PEG- and SDS-CuPani NPs are first incubated with KB cells for $24 \mathrm{~h}$ to evaluate the cytotoxicity. As shown in Fig. S6, $\uparrow$ the CuPani NSs and PEG-CuPani NPs exhibit relatively low cytotoxicity for KB cells. When the concentration is increased to $400 \mu \mathrm{g} \mathrm{mL} L^{-1}$, the relative cell viability is close to $90 \%$. In comparison, the SDSCuPani NPs show higher cytotoxicity which may result from the toxic component of SDS. But the slight cytotoxicity does not hinder the following cellular adhesion/internalization. Since proteins and cell membranes are mostly negatively charged in bio-systems, as well as cancerous cells, positively charged CuPani NSs can establish an effective electrostatic attraction towards cancerous cells, and quickly adhere onto cancerous cell membranes within a $10 \mathrm{~min}$ incubation (Fig. S7†). Further observed by fluorescent confocal microscopy, the CuPani NSs exhibit additional fast cellular internalization (Fig. S8†). This is also termed as nonspecific adhesion/internalization. The fast cellular adhesion/internalization is further analyzed among the CuPani NSs and NPs. After incubating with 293, KB and Hela cells for $10 \mathrm{~min}$, the adhered/internalized quantity is measured by optical density at $490 \mathrm{~nm}$. As shown in Fig. 3a, the adhered/ internalized quantities increase with the feeding concentration of NSs, and Hela cells show the best adhesion/internalization to NSs due to the strongest electrostatic attraction. The surface potentials are measured as $-18.1,-23.4,-29.1$ and $+33.0 \mathrm{mV}$ for 293, KB and Hela cells and CuPani NSs, respectively (Table $\mathrm{S} 1 \dagger)$. This consideration is further proven by adhering the CuPani NSs and Pani NSs without Cu(II) doping (+26.3 mV) on substrates separately modified with $-\mathrm{NH}_{2}$ and $-\mathrm{COOH}$ (Fig. S9†). Scanning electron microscopy (SEM) observations
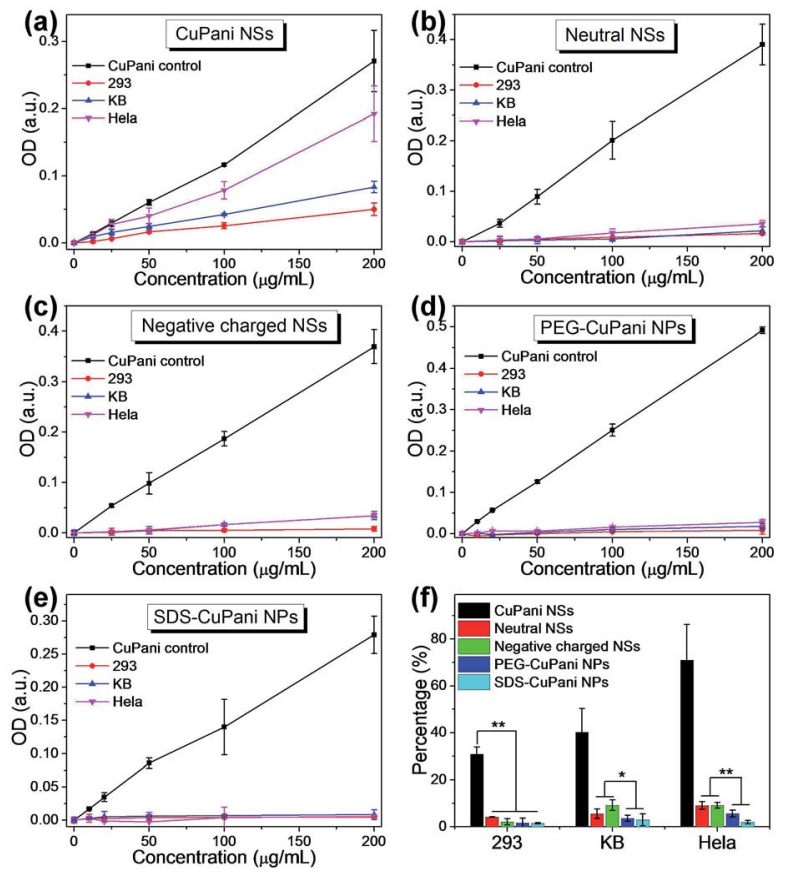

Fig. 3 Nonspecific cellular adhesion/internalization of CuPani NSs (a), neutral NSs (b), negatively charged NSs (c) and PEG-CuPani (d) and SDS-CuPani (e) NPs for 293, KB, and Hela cells. The CuPani control represents the $O D$ values at the feeding concentration. (f) The percentages of adhered/internalized CuPani NSs and NPs at a concentration of $200 \mu \mathrm{g} \mathrm{mL}^{-1}$. 
reveal that both Pani and CuPani NSs prefer to adhere on the substrates with $-\mathrm{COOH}$ (Fig. S9 $\dagger$ ). Moreover, the adhesion of the CuPani NSs is 2.3 times tenser than that of the Pani NSs because of the higher surface potential after the $\mathrm{Cu}$ (II) doping (Table S1 $\dagger$ ), confirming that the fast cellular adhesion/ internalization mainly depends on the intensity of the electrostatic attraction. In contrast, neutral $(-5.8 \mathrm{mV})$ and negatively charged $(-20.5 \mathrm{mV})$ CuPani NSs exhibit much lower adhesion/ internalization, while PEG-CuPani $(-5.6 \mathrm{mV})$ and SDS-CuPani $(-20.1 \mathrm{mV})$ NPs can hardly adhere onto cell membranes (Fig. 3b-e). The adhered percentages of CuPani are further compared at an incubating concentration of $200 \mu \mathrm{g} \mathrm{mL}$ (Fig. 3f), and the results indicate that only electrostatic attraction can trigger fast cellular adhesion/internalization. The cellular adhesion/internalization rate depends on the intensity of electrostatic attraction, and the CuPani NSs show the best nonspecific adhesion/internalization towards Hela cells. Besides, comparing neutral and negatively charged CuPani NSs with NPs, the shuttle-like morphology with an ultra-small dimension contributes more to the cellular adhesion/ internalization than other morphologies.

\subsection{Tumor uptake in vivo}

The electrostatic attraction and shuttle-like morphology may also benefit the tumor uptake by actively retaining the NSs and NPs in tumor tissues, and the CuPani NSs and NPs are further tested in tumor models in vivo. Mice are planted with $\mathrm{KB}$ and Hela tumors simultaneously (Fig. 4a). Firstly, the short term safety is investigated by liver and renal function tests for the CuPani NSs and NPs $24 \mathrm{~h}$ post intravenous (i.v.) injection. As shown in Fig. 5, the CuPani NSs and NPs hardly show any influence on the liver and renal functions compared to the age matched healthy mice. Then the mice are examined by MRI under a 1.5 T magnetic field. As shown in Fig. 4b, both KB and Hela tumors have low MRI signals in green without an injection. After injection with the CuPani NSs, the Hela tumors exhibit a stronger MRI signal in red than the KB tumors in green and yellow due to the different retention capability of the tumor tissues (Fig. 4c). Furthermore, because of the different retention capability in the tumor tissues motivated by electrostatic attraction, the Hela tumors exhibit a stronger MRI signal in red than the KB tumors in green and yellow, which is on average 2.9 times that of the $\mathrm{KB}$ tumors as determined from the relative
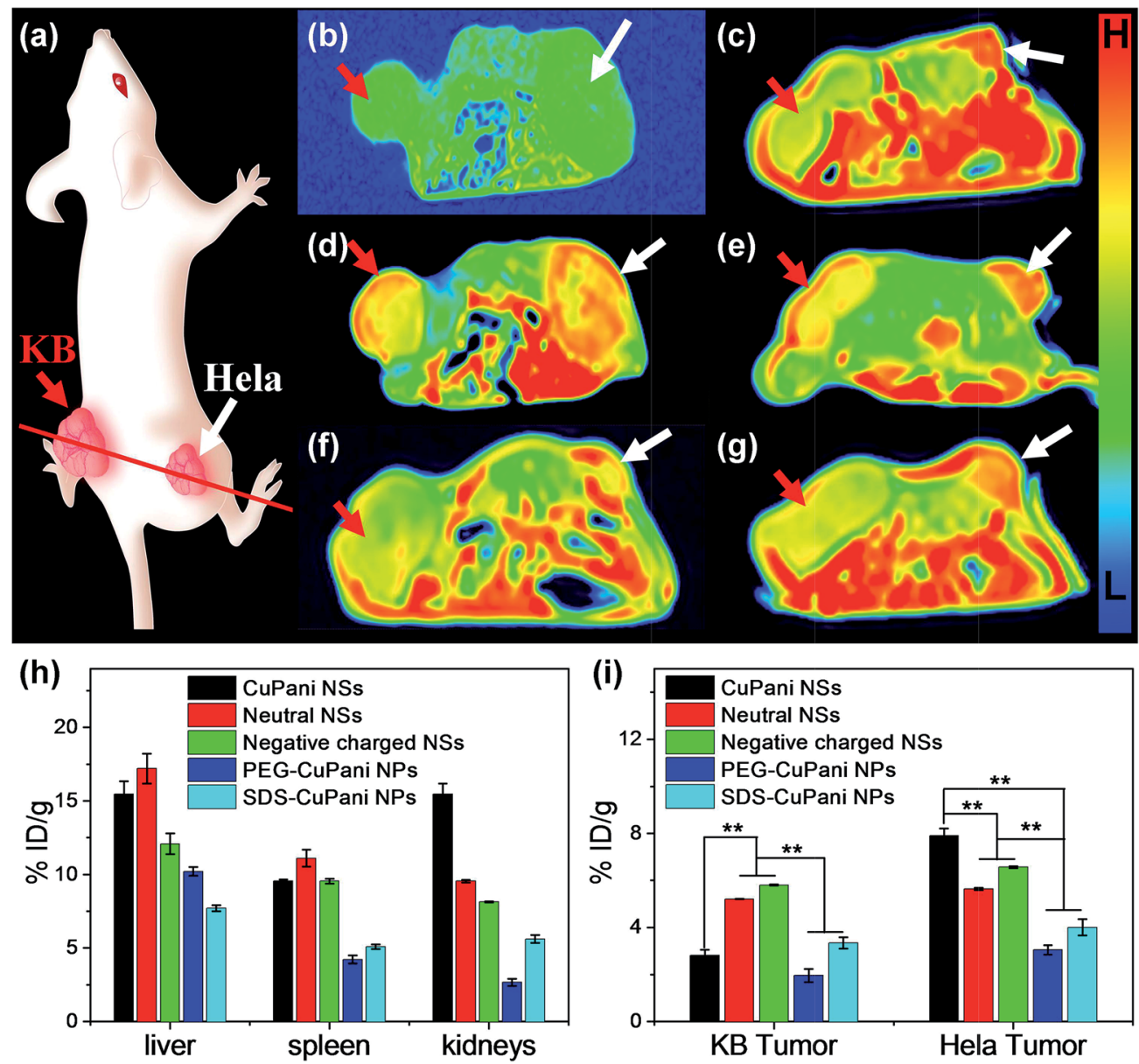

Fig. 4 The tumor diagnosis is tested in mice bearing KB and Hela tumors simultaneously (a). Representative MRI images taken $24 \mathrm{~h}$ post i.v. injection of saline (b), CuPani NSs (c), neutral NSs (d), negatively charged NSs (e) and PEG-CuPani (f) and SDS-CuPani (g) NPs. The color bar stands for the MRI intensity from low (blue) to high (red). The bio-distribution in vital organs (h) and accurate tumor uptake rates (i) were further determined at a content of $\mathrm{Cu}$ by ICP-AES. 

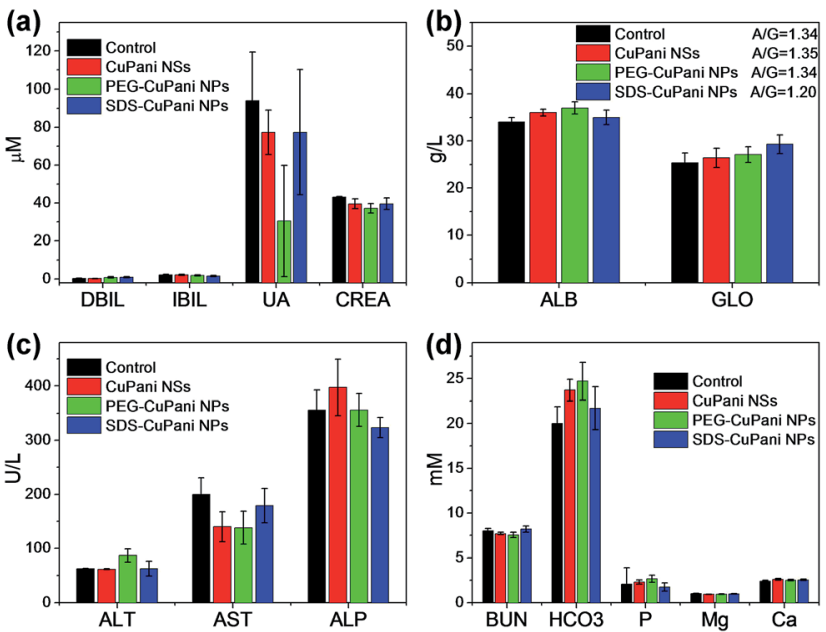

Fig. 5 Liver and renal functions of CuPani NSs and PEG-CuPani and SDS-CuPani NPs tested $24 \mathrm{~h}$ post i.v. injection. All of the parameters are in a normal scale compared to the age-matched healthy mouse (the control) including direct bilirubin (DBIL), indirect bilirubin (IBIL), albumin (ALB), globulin (GLO), alanine aminotransferase (ALT), aspartate transaminase (AST), alkaline phosphatase (ALP), blood urea nitrogen (BUN), content of dicarbonate $\left(\mathrm{HCO}_{3}\right)$, serum phosphorus $(\mathrm{P})$, serum magnesium (Mg), total calcium (Ca), uric acid (UA) and creatinine (CREA). A/G represents the ratio of ALB to GLO

MRI signals. The tumor uptake rate is determined as 7.9 and 2.8 percent of the injected dose per gram tissue (\% ID per $g$ ) for Hela and KB tumors by ICP-AES, respectively (Fig. 4i).

It should be mentioned that the CuPani NSs show a poor stealth effect due to their positively charged surface without any modification. Their visibility to the immune system leads to fast clearance out of the blood (Fig. 6a and d) and the half-life is only $3.2 \pm 0.4 \mathrm{~h}$ (Fig. 6c). Dramatic accumulation in vital organs such as the liver (15.5\% ID per $\mathrm{g}$ ) and kidneys (15.8\% ID per $\mathrm{g}$ ) is also observed in the bio-distributions (Fig. 4h, 7 and $8 \mathrm{a}$ and b). Nevertheless, the uptake by Hela tumors remains $7.9 \%$ ID per $g$, indicating that effective electrostatic attraction driven retention is the dominant factor for a high tumor uptake rate, especially for nanomaterials with a short blood circulation time. As for the $\mathrm{KB}$ tumors, the uptake rate is only $2.8 \%$ ID per $\mathrm{g}$, indicating that electrostatic attraction with $\mathrm{KB}$ tumor tissues is insufficient when there is competition for capture by the immune system. Based on the difference in electrostatic attraction, the CuPani NSs are capable of achieving nonspecific tumor recognition between KB and Hela tumors.

In order to improve the uptake of $\mathrm{KB}$ tumors, surface modification is required for the CuPani NSs. Neutral and negatively charged CuPani NSs are modified post i.v. injection. Compared to the mice injected with the CuPani NSs, MRI images show an enhanced uptake by KB tumors and a smaller difference between the KB and Hela tumors (Fig. $4 \mathrm{~d}$ and e). The $\mathrm{KB}$ tumor uptake rates are determined as 5.2 and 5.8\% ID per $\mathrm{g}$ for neutral and negatively charged CuPani NSs, respectively (Fig. 4i). The enhanced KB tumor uptake contributes to the improved invisibility against the immune system after surface adjustment, which is further confirmed by the blood
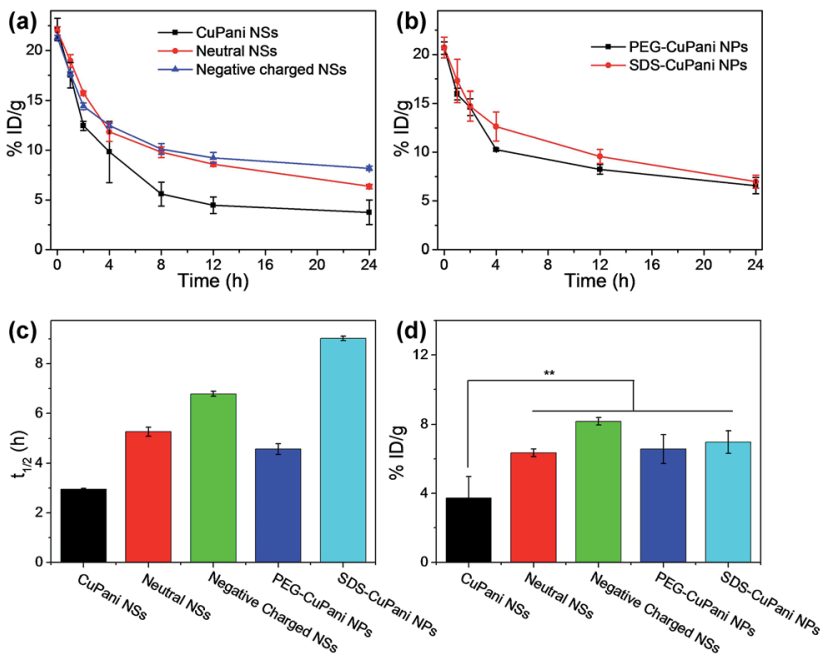

Fig. 6 The blood circulation of CuPani NSs, neutral NSs and negatively charged NSs (a) and PEG-CuPani and SDS-CuPani NPs (b) as tested in mice bearing double tumors of $\mathrm{KB}$ and Hela after i.v. injection. The half lives (c) and blood concentration $24 \mathrm{~h}$ post i.v. injection (d) of CuPani NSs, neutral NSs, negatively charged NSs and PEG-CuPani and SDSCuPani NPs. All of these results are based on samples determined by ICP-AES

circulation. As shown in Fig. 6, neutral and negatively charged NSs exhibit a much slower consumption during blood circulation, and the half-life is calculated as $5.3 \pm 0.2$ and $6.8 \pm 0.1 \mathrm{~h}$ (Fig. 6a and c). These evidences indicate that both neutral and negatively charged NSs possess a better stealth effect than positively charged CuPani NSs, which leads to reduced accumulation in the liver and kidneys (Fig. 4h). However, the uptake rate by Hela tumors decreases to 5.6 and $6.6 \%$ ID per $g$ for neutral and negatively charged NSs, respectively, indicating that the accumulation of neutral and negatively charged NSs in tumors is not solely dependent on the improved stealth effect. The shuttle-like morphology may also contribute to the tumor uptake.

The shuttle-like morphology may also benefit tumor tissue retention by establishing effective cellular internalization, and the high accumulation in kidneys may be contributed to by the ultra-small dimension of the CuPani NSs (Fig. $4 \mathrm{~h}$ and $8 \mathrm{~b}-\mathrm{d}$ ). To
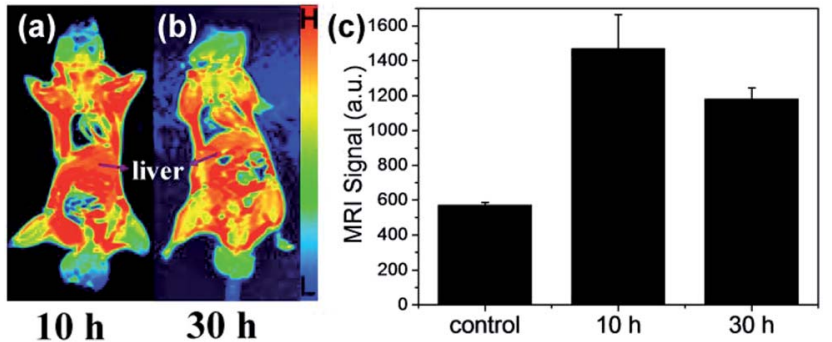

Fig. $7 \mathrm{MRI}$ tests are carried out to monitor the bio-distribution of the CuPani NSs in the mice at 10 (a) and $30 \mathrm{~h}$ (b) post i.v. injection. The color bar stands for the MRI intensity from low (blue) to high (red). The $M R I$ intensities in the liver are recorded in (c), while a healthy mouse is used as the control. 


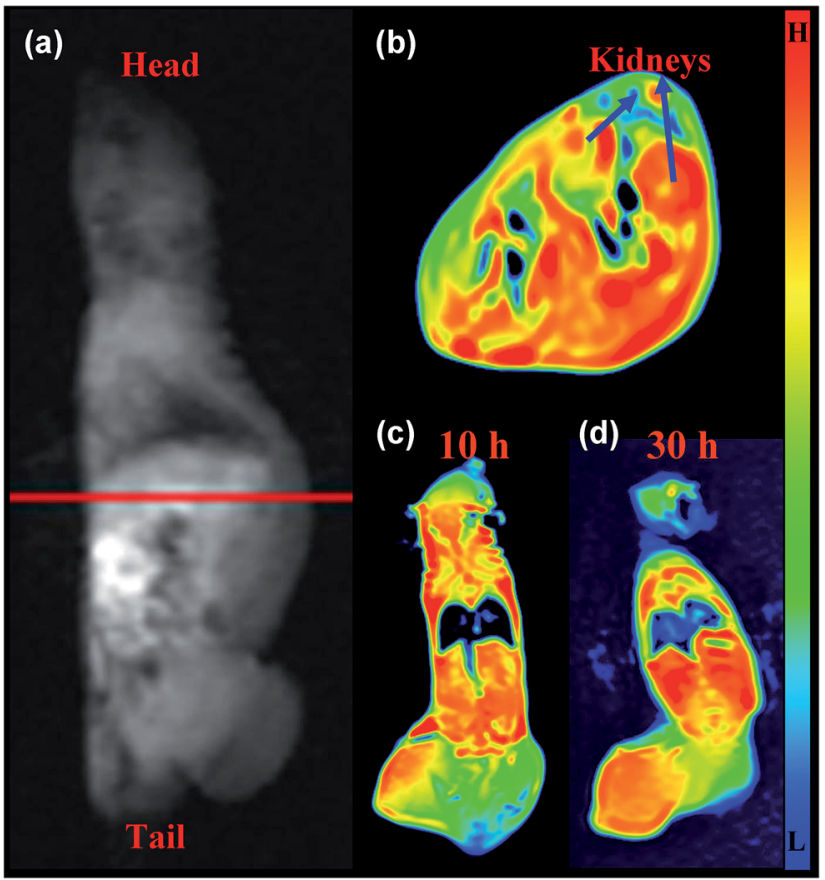

Fig. 8 The accumulation of CuPani NSs in the kidneys $24 \mathrm{~h}$ after i.v injection revealed by MRI in black and white (a) as well as in color (b). The accumulation in the kidneys is also compared at $10 \mathrm{~h}$ (c) and $30 \mathrm{~h}$ (d) post i.v. injection, and the MRI signals in the kidneys are $1176 \pm 87$ and $1308 \pm 29$, respectively. The color bar stands for the MRI intensity from low (blue) to high (red).

reveal the contribution from the shuttle-like morphology, PEGCuPani and SDS-CuPani NPs are also i.v. injected to evaluate the tumor uptake. As shown in Fig. $4 \mathrm{f}$ and $\mathrm{g}$, both PEG-CuPani and SDS-CuPani NPs can accumulate in the tumor area based on the EPR effect. Although the modification of stealthy polymer PEG and negatively charged SDS on CuPani NPs are in favor to reduce the nonspecific uptake, and the consumption in blood is slow as the respective half-lives are $4.6 \pm 0.2$ and $9.0 \pm 0.1 \mathrm{~h}$ (Fig. $4 \mathrm{~h}$ and $6 \mathrm{~b}-\mathrm{d}$ ), the uptake rates for $\mathrm{KB}$ and Hela tumors are only 1.9 and $3.0 \%$ ID per $\mathrm{g}$ for PEG-CuPani NPs, and 3.3 and 4.0\% ID per $\mathrm{g}$ for SDS-CuPani NPs, respectively (Fig. 4f, $\mathrm{g}$ and i). Compared to the PEG-CuPani NPs with neutral NSs, as well as the SDS-CuPani NPs with negatively charged NSs, the nonshuttle-like morphologies circulate for an equal or longer time than the NSs, but the tumor uptake rate is far lower than that of the NSs, indicating that the shuttle-like morphology is superior to the other morphologies in tumor uptake. And for neutral and negatively charged NSs, the shuttle-like morphology is the main contributor to tumor uptake other than the improved stealth effect. This also explains why for neutral and negatively charged NSs the uptake for KB tumors is significantly enhanced while the uptake for Hela tumors decreases slightly.

Although positively charged CuPani NSs show poor invisibility to the immune system, they may show another advantage in bio-safety due to their fast clearance. As shown in the MRI images $10 \mathrm{~h}$ post i.v. injection (Fig. 7a), a dramatic accumulation in the liver is observed and the intensity of the MRI signal rises up to $\sim 1500$. Compared with the MRI test $30 \mathrm{~h}$ post injection (Fig. 7b and c), the MRI signal decreases to $\sim 1100$. Besides, a high dose of CuPani NSs also accumulates in the kidneys because of the small transverse dimension being about $14 \mathrm{~nm}$, but the renal filtration of NSs is slow and difficult because the longitudinal dimension is about $62 \mathrm{~nm}$ and rapid renal filtration requires the size of NPs to be below $5.5 \mathrm{~nm}$ (Fig. 2a and $8 \mathrm{c}$ and d). ${ }^{42}$ These results indicate that a possible metabolism route for the CuPani NSs is the hepatic and biliary route. The feasibility in metabolism leads to good safety in both the short and long term as mentioned in our previous report (Fig. 5). ${ }^{41}$

\section{Conclusions}

In summary, we demonstrate that positively charged CuPani NSs are capable of enhancing tumor uptake rate by being actively retained in tumor tissues due to the synergetic contributions from the electrostatic attraction and shuttle-like morphology. The difference in the surface potentials between Hela and KB tumors leads to a nonspecific tumor recognition of CuPani NSs, showing an uptake rate of $7.9 \%$ ID per $\mathrm{g}$ for Hela and $2.8 \%$ ID per $\mathrm{g}$ for KB tumors. When the stealth effect is improved, the shuttle-like structure of CuPani NSs also contributes to tumor uptake because of the one-dimensionallike morphology for effective cellular internalization. The synergetic enhancement of tumor uptake by controlling the surface charges and anisotropic morphology of nanomaterials provides a new pathway for designing novel nanodevices to perform tumor theranostics.

\section{Author contributions}

Min Lin and Shuwei Liu contributed equally to this paper. H. Z. proposed and supervised the project. H. Z., M. L., S. W. L., Y. L., W. Z. S., H. C. S. and B. Y. designed and performed the experiments and co-wrote the paper. D. D. W., S. Y. L., X. Z., R. G. and X. L. participated in most experiments. All authors have given approval to the final version of the manuscript.

\section{Conflicts of interest}

There are no conflicts to declare.

\section{Acknowledgements}

This work was supported by the NSFC (21374042, 51603084, 81372900, 51425303), JLU Science and Technology Innovative Research Team 2017TD-06 and the Special Project from MOST of China.

\section{References}

1 J. Conde, N. Oliva, Y. Zhang and N. Artzi, Nat. Mater., 2016, 15, 1128-1138.

2 S. Wilhelm, A. J. Tavares, Q. Dai, S. Ohta, J. Audet, H. F. Dvorak and W. C. W. Chan, Nat. Rev. Mater., 2016, 1, $1-12$. 
3 S. Wang, P. Huang and X. Chen, Adv. Mater., 2016, 28, 73407364.

4 M. H. Lee, J. L. Sessler and J. S. Kim, Acc. Chem. Res., 2015, 48, 2935-2946.

5 M. P. Melancon, M. Zhou and C. Li, Acc. Chem. Res., 2011, 44, 947-956.

6 M. E. Caldorera-Moore, W. B. Liechty and N. A. Peppas, Acc. Chem. Res., 2011, 44, 1061-1070.

7 Y. Lyu, Y. Fang, Q. Miao, X. Zhen, D. Ding and K. Pu, ACS Nano, 2016, 10, 4472-4481.

8 F. Tian, J. Conde, C. Bao, Y. Chen, J. Curtin and D. Cui, Biomaterials, 2016, 106, 87-97.

9 L. Rao, L.-L. Bu, B. Cai, J.-H. Xu, A. Li, W.-F. Zhang, Z.-J. Sun, S.-S. Guo, W. Liu, T.-H. Wang and X.-Z. Zhao, Adv. Mater., 2016, 28, 3460-3466.

10 G. Mikhaylov, D. Klimpel, N. Schaschke, U. Mikac, M. Vizovisek, M. Fonovic, V. Turk, B. Turk and O. Vasiljeva, Angew. Chem., Int. Ed., 2014, 53, 10077-10081.

11 Y.-L. Wu, N. Putcha, K. W. Ng, D. T. Leong, C. T. Lim, S. C. J. Loo and X. Chen, Acc. Chem. Res., 2013, 46, 782-791.

12 L. Zeng, Y. Pan, R. Zou, J. Zhang, Y. Tian, Z. Teng, S. Wang, W. Ren, X. Xiao, J. Zhang, L. Zhang, A. Li, G. Lu and A. Wu, Biomaterials, 2016, 103, 116-127.

13 J. Wang, P. Mi, G. Lin, Y. X. J. Wáng, G. Liu and X. Chen, Adv. Drug Delivery Rev., 2016, 104, 44-66.

14 K. Liu, Z. Nie, N. Zhao, W. Li, M. Rubinstein and E. Kumacheva, Science, 2010, 329, 197-200.

15 J. Song, P. Huang, H. Duan and X. Chen, Acc. Chem. Res., 2015, 48, 2506-2515.

16 T. Sun, Y. S. Zhang, B. Pang, D. C. Hyun, M. Yang and Y. Xia, Angew. Chem., Int. Ed., 2014, 53, 12320-12364.

17 Y. Li, J. Tang, L. He, Y. Liu, Y. Liu, C. Chen and Z. Tang, Adv. Mater., 2015, 27, 4075-4080.

18 M. Aioub and M. A. El-Sayed, J. Am. Chem. Soc., 2016, 138, 1258-1264.

19 C.-L. Zhang, K.-P. Lv, H.-P. Cong and S.-H. Yu, Small, 2012, 8, 648-653.

20 S. Schöttler, K. Landfester and V. Mailänder, Angew. Chem., Int. Ed., 2016, 55, 8806-8815.

21 B. Layek, T. Sadhukha and S. Prabha, Biomaterials, 2016, 88, 97-109.

22 A. A. Saie, M. Ray, M. Mahmoudi and V. M. Rotello, Cancer Treat. Res., 2015, 166, 245-273.

23 T. Yang, Y. Wang, H. Ke, Q. Wang, X. Lv, H. Wu, Y. Tang, X. Yang, C. Chen, Y. Zhao and H. Chen, Adv. Mater., 2016, 28, 5923-5930.

24 D. Hu, Z. Sheng, G. Gao, F. Siu, C. Liu, Q. Wan, P. Gong, H. Zheng, Y. Ma and L. Cai, Biomaterials, 2016, 93, 10-19.
25 G. Chen, L. Wang, T. Cordie, C. Vokoun, K. W. Eliceiri and S. Gong, Biomaterials, 2015, 47, 41-50.

26 S. Schöttler, G. Becker, S. Winzen, T. Steinbach, K. Mohr, K. Landfester, V. Mailänder and F. R. Wurm, Nat. Nanotechnol., 2016, 11, 372-377.

27 Y. Chen, H. Han, H. Tong, T. Chen, H. Wang, J. Ji and Q. Jin, ACS Appl. Mater. Interfaces, 2016, 8, 21185-21192.

28 L. Cui, Q. Lin, C. S. Jin, W. Jiang, H. Huang, L. Ding, N. Muhanna, J. C. Irish, F. Wang, J. Chen and G. Zheng, ACS Nano, 2015, 9, 4484-4495.

29 L. Li, S. Fu, C. Chen, X. Wang, C. Fu, S. Wang, W. Guo, X. Yu, X. Zhang, Z. Liu, J. Qiu and H. Liu, ACS Nano, 2016, 10, 70947105.

30 T. Wang, D. Wang, H. Yu, M. Wang, J. Liu, B. Feng, F. Zhou, Q. Yin, Z. Zhang, Y. Huang and Y. Li, ACS Nano, 2016, 10, 3496-3508.

31 Y. Ma, Z. Wang, M. Zhang, Z. Han, D. Chen, Q. Zhu, W. Gao, Z. Qian and Y. Gu, Angew. Chem., Int. Ed., 2016, 55, 33043308.

32 Y. Chen, D. Ye, M. Wu, H. Chen, L. Zhang, J. Shi and L. Wang, Adv. Mater., 2014, 26, 7019-7026.

33 Y. Dou, Y. Guo, X. Li, X. Li, S. Wang, L. Wang, G. Lv, X. Zhang, H. Wang, X. Gong and J. Chang, ACS Nano, 2016, 10, 2536-2548.

34 M. Yang, Q. Fan, R. Zhang, K. Cheng, J. Yan, D. Pan, X. Ma, A. Lu and Z. Cheng, Biomaterials, 2015, 69, 30-37.

35 X. Zhong, K. Yang, Z. Dong, X. Yi, Y. Wang, C. Ge, Y. Zhao and Z. Liu, Adv. Funct. Mater., 2015, 25, 7327-7336.

36 M. Lin, D. Wang, S. Li, Q. Tang, S. Liu, R. Ge, Y. Liu, D. Zhang, H. Sun, H. Zhang and B. Yang, Biomaterials, 2016, 104, 213-222.

37 L. S. Dake, D. E. King and A. W. Czanderna, Solid State Sci., 2000, 2, 781-789.

38 Z. Zhang, X. Zhang, T. Zheng, H. Yu and Q. Liu, J. Mol. Struct., 1999, 478, 23-27.

39 C. D. Wagner, J. F. Moulder, L. E. Davis and W. M. Riggs, Handbook of X-ray Photoelectron Spectroscopy, PerkingElmer Corporation, Physical Electronics Division, USA, 1979.

40 S. Suzuki, T. Kosaka, M. Saito, H. Inoue, Y. Waseda, E. Matsubara and M. Oku, Scr. Mater., 1997, 36, 841-845.

41 J. Yang, J. Choi, D. Bang, E. Kim, E.-K. Lim, H. Park, J.-S. Suh, K. Lee, K.-H. Yoo, E.-K. Kim, Y.-M. Huh and S. Haam, Angew. Chem., Int. Ed., 2011, 50, 441-444.

42 H. S. Choi, W. Liu, P. Misra, E. Tanaka, J. P. Zimmer, B. I. Ipe, M. G. Bawendi and J. V. Frangioni, Nat. Biotechnol., 2007, 25, 1165-1170. 